\title{
Information query system optimization design in the construction of computer professional
}

\author{
Liu chang jun \\ Sichuan Information Techology College 628040
}

Keywords: information query; fuzzy sets; heterogeneous bipolar information

\begin{abstract}
In the study of information query system optimization design in the construction of computer professional, with the current algorithms to process the optimal design for query system, the user is not familiar with what is allowed or not, or unable to enumerate all (not) allowed values of information, resulting in low query efficiency and large error. For this, an information query system optimization design method in the construction of computer professional based on improved fuzzy algorithm is proposed. The algorithm first analyze personalized development of query information through the perspective of computer information filtering, through similarity matching of user demand and information content to filter and information independent from user needs, fused with fuzzy algorithm, fuzzy set is utilized to build heterogeneous bipolar information query satisfaction model framework composed of an independent satisfaction and dissatisfaction. On this basis, information query system optimization design in the construction of computer professional is achieved. Simulation results show that the information query system optimization design method in the construction of computer professional based on improved fuzzy algorithm can effectively improve the accuracy and efficiency of query.
\end{abstract}

\section{Introduction}

In recent years, science and technology are developing continually, the application of computer has been more and more wide [1,2,3]. The amount of information is growing rapidly, at the same time, people are also gradually increasing requirements for the computer information system $[4,5,6]$. Optimizing the design of information query system in the construction of computer professional, can effectively meet the needs of the people, attracts the attention of a lot of experts and scholars $[7,8,9]$.

Currently, the mainstream query system optimization design algorithm in the construction of computer professional mainly includes multi constrained model algorithm, association rules algorithm and neural network algorithm. Among them, the commonly used is information system design method based on multi constrained model algorithm. But when this algorithms is used to process the optimal design for query system, the user is not familiar with what is allowed or not, or unable to enumerate all (not) allowed values of information, resulting in low query efficiency and large error.

\section{Description of personality development of computer information query system based on information filtering}

Personalized query system of computer is to achieve the purpose of recommending by collecting and analyzing users' information to learn the user's interest and behavior. From the perspective of information filtering, the personalized development of computer network information query system has the following 3 forms:

(1) Personalization of designed content filtering of the computer information query system

Personalized information query system based on content filtering is a filtering form from content and user model point of view, it refers that the computer network collects useful information and information users interested in from a large number of document or information, and the information is classified, so as to establish the user module.

(2) Personalization of collaborative filtering of computer information query system design 
For computer users have needs for common information or similar preference, the computer information query system according to the information similarity in user demand module to classify and cluster the user groups, so as to provide query request of users have similar interest with query result which meets their needs best, and filter the resources content independent from groups similarity.

(3) Personalization of hybrid filtering of computer information query system design

Information filtering of computer information query system including two kinds of system structure, collaborative filtering and content filtering, the combination of these two kinds of filtering forms can make the computer user to find the most valuable information resources.

\section{Design principle of information query system optimization method in the construction of in computer professional}

\section{1 user model based on information filtering}

During the process of information query system optimization design in the construction of computer professional, from the group perspective to group and cluster computer users according to the computer users' preference and, data collection, refining and storage in computer information query system are processed according to the user's preference. In the process of the computer information query system optimization design, implementation can be divided into 3 stages:

(1) Calculation stage of information relevancy in the process of information query system optimization design in the construction of computer professional

The computer system calculates the relevancy for the collected document or information through the matching degree of computer and the computer user's needs similarity, and stores the results into the search list, so as to make preparatory work for information filtering of information query system.

(2) Information content filtering stage in the optimization design process of information query system in the construction of computer major

The stage of information filtering of computer information query system is defined as to find the document or information satisfied to specific user requirements from a large flow.

(3) Filtering result preservation and interactive stage in the optimization design process of information query system in the construction of computer major

3.2 The realization of optimization design method for information query system in the construction of computer major

Integration of fuzzy algorithm, using fuzzy set construct a heterogeneous bipolar information query satisfaction model framework consisted of a separate satisfaction and dissatisfaction, on this basis, to realize the optimization design of information query system in the construction of computer major.

In the optimal design process of the information query system in the construction of computer science major, bipolar satisfaction mainly used for expressing satisfaction and dissatisfaction, the value of it can be represented as following:

$$
(s, \mathrm{~d}), \mathrm{s}, \mathrm{d} \in[0,1]
$$

Among them, $s$ is degree of satisfaction, $d$ is degree of non-satisfaction.

In the optimal design process of the information query system in the construction of computer science major, the value range of $s$ and $d$ is $[0,1]$, they are independent of each other, and express satisfaction and dissatisfaction. $(1,0)$ indicates "completely satisfied, it has no dissatisfied, $(0,1)$ indicates " it has no dissatisfied, completely dissatisfied ", making the bipolar satisfaction is $\mathrm{ds}$, then it has the following formula:

$$
\mathrm{d} s=|(s, d)| s, d \in[0,1]
$$

It can be concluded from the above formula, bipolar satisfaction is directly related to the fuzzy set.

In the optimization design process of information query system in the construction of computer 
major, bipolar query conditions can specify the satisfaction and dissatisfaction with a range of $[0,1]$ in the domain for each value. Therefore, in the basic query selection condition, the heterogeneous bipolar for expression of computer user preferences can formally make modeling by bipolar extension of fuzzy set. While, fuzzy sets meet this requirement. But in the definition of fuzzy sets, the presence of the consistency constraints $0 \leq t v(u) \leq 1-f v(u) \leq 1$, affects the independence of satisfaction and satisfaction value in heterogeneous bipolar information. In order to fully reflect the computer user preferences in the real world, in the optimization design process of information query system in the construction of computer major, the constraint conditions of fuzzy set can be made relaxation processing, which means that the $0 \leq t v(u) \leq 1-f v(u) \leq 1$ does not have to be set up.

In the optimal design process of referral information system in the construction of computer science, considering a basic condition $C$ aimed at property $A$, the domain of discourse $d{ } m_{A}$ of attribute $A$ indicates the user's preference related to the tuple value of attribute $A$. Therefore, the most simple generic form of condition $C$ can use fuzzy set to make modeling:

$$
C=\sum_{i=1}^{n}\left(t_{c}\left(x_{i}\right), f_{c}\left(x_{i}\right)\right) / x_{i} \in \operatorname{dom}_{A}
$$

Among them, the membership function $t_{c}$ defined the positive preference of computer users, that is to say, the membership $t_{c}(x)$ related to threshold $x_{i} \in \operatorname{dom}_{A}$ represent the degree of that $x$ meets the attributes $A$, non-membership degree function $f c$ defined a negative preference of users, that is to say, the membership $t_{c}(x)$ related to threshold $x_{i} \in \operatorname{dom}_{A}$ represent the degree of that $x$ cannot meet the attributes $A$.

\section{Simulation Experiment}

In order to prove the effectiveness of the information query system optimization design method in construction of computer professional based on improved fuzzy algorithm, there is the need for experiment, information query system optimization design platform in the construction of computer professional is built in the virtual Matlab7.0 environment, using the traditional method and the improved method for query optimization design of information system in the construction of computer professional, information query accuracy of systems designed with different algorithm are compared, the comparison results are shown in table 1 .

Table 1 Comparison of accuracy degree of different algorithm

\begin{tabular}{lllll}
\hline the number of experiments & $\begin{array}{c}\text { Accuracy of } \\
\text { algorithm }\end{array}(\%)$ & $\begin{array}{c}\text { Accuracy of } \\
\text { algorithm }\end{array}$ & improved \\
\hline 1 & 98 & 87 & \\
2 & 98 & 87 & \\
3 & 96 & 85 & \\
4 & 97 & 86 & \\
5 & 95 & 84 & \\
6 & 96 & 85 & \\
7 & 97 & 86 & \\
8 & 95 & 84 & \\
9 & 96 & 85 & \\
10 & 98 & 87 & \\
\hline
\end{tabular}

With the system designed by different algorithm to compare Information query time, the results shown in figure 1. 


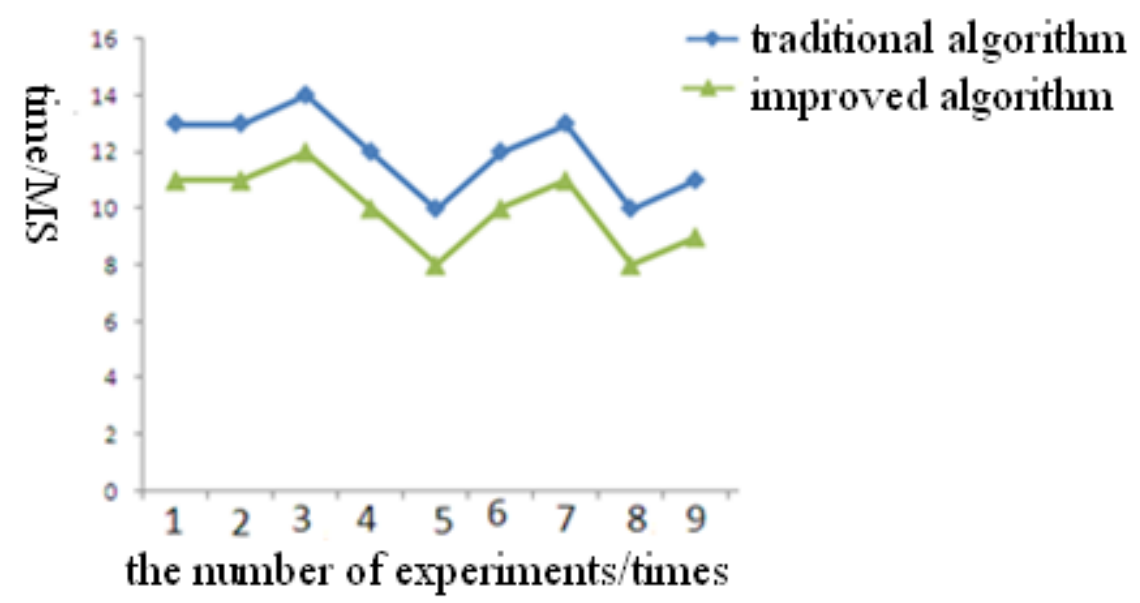

Figure 1 Information query time comparison of different algorithm design system

The experimental results show that the information query system optimization design method in the construction of computer professional based on improved fuzzy algorithm is practical, can satisfy the application demand of information query system optimization design in the construction of computer professional.

\section{Conclusions}

With the current algorithms to process the optimal design for query system, the user is not familiar with what is allowed or not, or unable to enumerate all (not) allowed values of information, resulting in low query efficiency and large error. For this, an information query system optimization design method in the construction of computer professional based on improved fuzzy algorithm is proposed. The algorithm first analyze personalized development of query information through the perspective of computer information filtering, through similarity matching of user demand and information content to filter and information independent from user needs, fused with fuzzy algorithm, fuzzy set is utilized to build heterogeneous bipolar information query satisfaction model framework composed of an independent satisfaction and dissatisfaction. On this basis, information query system optimization design in the construction of computer professional is achieved. Simulation results show that the information query system optimization design method in the construction of computer professional based on improved fuzzy algorithm can effectively improve the accuracy and efficiency of query.

\section{References}

[1] Li Yongli. Design of virtualization technology in meteorological service information system [J]. Computer CD Software and Applications. 2013.5:31-32.

[2] Shi Yun, Zhang Lisun, Xu Jun. Discussion on the construction of water conservancy information data center [J]. Engineering construction and design.2013.8:140-143.

[3] Hui Li,Wu Ling, Yu Liping. Optimization and improvement of hardware curriculum system construction in computer major [J]. Education in Heilongjiang: higher educational research and evaluation.2013.4:45-46.

[4] Bi Kejun, Zhang Weiguo, Zhang Meng, Liu Baoning. Optimal design of longitudinal control stability system based on model reference [J]. Computer simulation.2013.2:76-79.

[5] Chen Qian. The application of computer simulation in airport planning and design [J]. Chinese science and technology.2013.17:107-107.

[6] Huang Jianhua. Optimization design of automated stereoscopic warehouse control system based on PLC [J]. Industrial control computer. 2013.11.155-156.

[7] Zhan Xuan, Lv Xiaojun, Liu Weiping, Feng Wenxue. Research and implementation of remote 
voice module in passenger service integrated management platform [J]. Railway computer application.2014.4:27-29.

[8] Zhou Wenqiong, Wang Leqiu, Xie Mei, Shang Min. The system design and implementation of the online financial search based on the ASP.NETMVC framework [J]. Software Guided. 2013.1:62-63.

[9] Zhuang Jun, Qiang Chunxia. Analysis and Design of Shop Drawing Censoring System [J]. Intelligent computer and application of.2013.2:74-76. 